\title{
Effects of enteral nutrition with parenteral glutamine supplementation on the immunological function in septic rats
}

\author{
Jun Fan ${ }^{1 *}$, Lidong Wu ${ }^{1}$, Guoping $\mathrm{Li}^{1}$, Shaoyu Tao ${ }^{1}$, Zhiyong Sheng ${ }^{1}$, Qingyan Meng ${ }^{2}$, Fengxin $\mathrm{Li}^{1}$, \\ Lijuan $\mathrm{Yu}^{1}$ and $\mathrm{Li} \mathrm{Li}^{1}$ \\ ${ }^{1}$ Department of Emergency and Critical Care Medicine, The Second Affiliated Hospital of Nanchang University, \\ 1 Minde Road, Nanchang 330006, Jiangxi, People's Republic of China \\ ${ }^{2}$ Department of Burns, The Northern Hospital, 83 Wenhua Road, Shenyang 110015, Liaoning, People's Republic of China
}

(Submitted 11 July 2014 - Final revision received 16 February 2015 - Accepted 10 March 2015 - First published online 21 April 2015)

\section{Abstract}

The aim of the present study was to investigate the effects of enteral nutrition (EN) with parenteral glutamine (GLN) supplementation on inflammatory response, lymphatic organ apoptosis, immunological function and survival in septic rats by caecal ligation and puncture (CLP). Male rats were randomly assigned into two experimental groups and two sham CLP control groups ( $n 10$ per group). After CLP or sham CLP model and nutrition programme were completed, the GLN concentrations of plasma and tissues and several indices of immunological function including serum Ig content, circulating lymphocyte number, the CD4:CD8 ratio, the neutrophil phagocytosis index (NPI), the organ index and apoptosis of thymus and spleen, and plasma cytokine levels were determined. Moreover, the survival in septic rats was observed. The results revealed that EN with parenteral GLN supplementation remarkably increased the GLN concentrations of plasma and tissues, serum Ig content, the circulating lymphocyte number, the CD4:CD8 ratio, the indexes of thymus and spleen, NPI and survival compared with the control group $(P<0.05)$. In contrast, the apoptosis of thymus and spleen and the levels of TNF- $\alpha$, IL-1 $\beta$ and IL- 6 in plasma were obviously decreased compared with the control group $(P<0 \cdot 05)$. These results show that EN with parenteral GLN supplementation diminished the release of inflammatory cytokines, attenuated lymphatic organ apoptosis, enhanced the immunological function and improved survival in septic rats.

\section{Key words: Enteral nutrition: Glutamine: Immunity: Cytokines: Sepsis}

Severe sepsis is still one of the leading causes of death in critically ill patients ${ }^{(1-4)}$. The precise pathophysiological mechanisms by which sepsis develops are not completely understood. However, experimental and clinical studies have revealed that immune dysfunction during sepsis plays a crucial role in the outcome ${ }^{(2)}$. In the early phase of sepsis, the immune response may be characterised by the release of pro-inflammatory mediators, and most patients may survive. In the late phase, the patients often demonstrate signs of anti-inflammatory immune suppression such as failing to eradicate invading pathogens and being susceptible to opportunistic organisms ${ }^{(1-4)}$. Because of the mortality of severe sepsis, until now, great efforts have been undertaken to develop an immune-modulating therapy to improve the outcome of sepsis. A better therapeutic strategy in the modulation of immune dysfunction of sepsis might control non-pathogen-specific inflammatory response to avoid tissue damage as well as enhance anti-infectious immune response.
Since nutritional support may provide all kinds of macro- and micronutrients, including carbohydrate, fat, protein, vitamin and trace elements, for critically ill patients to meet the daily requirements and incremental expenditure due to stressinduced hypermetabolic and hypercatabolic states, it has become an integral and indispensable part of the treatment of the critically ill patients ${ }^{(5)}$. Because of its obvious advantages (maintaining gut integrity and protecting against bacterial translocation, lower cost, better physiology and lower complication rate), enteral nutrition (EN) is viewed as the first line of feeding of the critically ill patients ${ }^{(6)}$. In critically ill septic patients, a energy and protein delivery closer to recommended amounts by EN in the early phase of intensive care unit stay has been associated with a more favourable outcome ${ }^{(7)}$. With the development of modern nutritional therapeutic theory and technique, it has been recognised that immunonutrition containing specific nutrients such as glutamine (GLN), arginine, RNA nucleotides, $n$ - 3 fish oil and others may attain the aim of

Abbreviations: AA, glutamine-free amino acid; CLP, caecal ligation and puncture; EN, enteral nutrition; GLN, glutamine; NPI, neutrophil phagocytosis index; TUNEL, terminal deoxynucleotidyl transferase dUTP nick end labelling. 
enhancing host immune function, modulating inflammatory response and improving protein synthesis ${ }^{(8)}$. One of the more highly studied nutrients for this purpose is the amino acid GLN.

GLN, traditionally considered to be a non-essential amino acid, is now considered as 'conditionally essential' amino acid during inflammatory and stress states ${ }^{(9)}$. GLN is an important and unique amino acid, with intermediary metabolism roles and others ${ }^{(10)}$. Numerous laboratory and clinical studies have demonstrated that GLN could modulate inflammatory response and stimulate immune function ${ }^{(11-25)}$. It has been widely applied in critically ill patients. However, recently, a meta-analysis of randomised clinical trials has proclaimed that GLN supplementation in critically ill patients has no significant effects on infections, length of stay and mortality ${ }^{(26)}$. Further, Heyland et al. ${ }^{(27)}$ even reported that GLN supplementation has been associated with an increase in mortality among critically ill patients, whereas Wischmeyer et $a l^{(28)}$ strongly supported that parenteral GLN supplementation in critically ill patients could improve survival rate and decrease infectious complications, costs and hospital length-of-stay. These conflicting and confusing results may bring about the controversy over GLN supplementation in critically ill patients. Although the present data are contradictory, the potential benefit of parenteral GLN supplementation has been one of the most heavily investigated issues of nutritional interventions in critical care medicine. Further exploration of the mechanisms of GLN immunomodulating role appears warranted and necessary. The objective of the present study was to investigate the impact of $\mathrm{EN}$ with parenteral GLN supplementation on inflammatory response, lymphatic organ apoptosis, immunological function and survival in septic rats by caecal ligation and puncture (CLP).

\section{Materials and methods \\ Animals preparation}

Male Sprague-Dawley rats weighing 250-300 g were used in this experiment. All rats were housed in stainless steel cages maintained in a temperature- and humidity-controlled room. Rats were allowed free access to a rodent chow and water for a 7-d acclimatisation period. All procedures of the present study were in accordance with the guide for the care and the use of laboratory animals published by the Ministry of Science and Technology of China, and were approved by the Ethics Committee of our institute.

\section{Surgical procedure and grouping}

Male Sprague-Dawley rats were randomly assigned into four groups: a sham CLP+EN+GLN-free amino acid (AA) group ( $n$ 10); a sham CLP+EN+GLN group ( $n$ 10); a CLP+EN+AA group ( $n$ 10); a CLP+EN+GLN group ( $n$ 10). Sepsis was induced by CLP. Briefly, the rats were weighted and anaesthetised by intraperitoneal injection of $50 \mathrm{mg} / \mathrm{kg}$ body weight pentobarbital sodium, and were fixed in supine position, and the entire ventral surface was shaved, and the abdomen was sterilely opened through a $2 \mathrm{~cm}$ middle incision. The caecum was exposed. The distal $1 \mathrm{~cm}$ of the caecum was ligated with a 4-0 silk suture and perforated twice through-and-through with a 21-gauge needle. A small amount of faeces was extruded to ensure that the wound was patent. The caecum was placed back into the abdominal cavity, and the abdomen was closed with 4-0 silk suture in two layers. Immediately, the CLP rats received a $50 \mathrm{ml} / \mathrm{kg}$ body weight intraperitoneal injection of sterile $0.9 \%$ saline for fluid resuscitation. The rats in two sham CLP groups were treated in the same procedure as above. However, the caecum was exposed but not ligated or punctured, and then replaced. Animals were returned to their cages and allowed food and water ad libitum.

\section{Nutrition programme and sample collection}

Enteral feeding began $2 \mathrm{~h}$ after sham CLP or CLP and continued for $7 \mathrm{~d}$ (the enteral feedings were divided evenly two times per $\mathrm{d}$ ). All rats in each of the four groups received an identical EN solution as their base formula. As described in Table 1, convention EN solution provided, per litre, $6279 \mathrm{~kJ}$ $(1500 \mathrm{kcal}), 60 \mathrm{~g}$ protein, $185 \mathrm{~g}$ carbohydrate and $58.4 \mathrm{~g}$ fat. The supplied energy ratio of the EN solution was $49 \%$ carbohydrate, $35 \%$ fat and $16 \%$ protein, and the proportion of energy to N was 133:1. Simultaneously, GLN was administered as alanyl-glutamine dipeptide (Dipeptiven; Fresenius Kabi). The rats in the sham CLP+EN+GLN and the CLP+EN+GLN groups were given $0.35 \mathrm{~g}$ GLN/kg body weight per $\mathrm{d}$ once via a tail vein injection for $7 \mathrm{~d}$, whereas those in the sham $\mathrm{CLP}+\mathrm{EN}+\mathrm{AA}$ and the $\mathrm{CLP}+\mathrm{EN}+\mathrm{AA}$ groups were administered isoenergetic/isonitrogenous GLN-free amino acid solution for comparison. There was isonitrogenous, isovolumic and isoenergetic intake in four groups. All rats among four groups were given $1213 \mathrm{~kJ}(290 \mathrm{kcal}) / \mathrm{kg}$ body weight per $d$. A quarter of the daily requirement of energy was supplied within the first $24 \mathrm{~h}$, and a half in the second $24 \mathrm{~h}$. After the 2nd day, the full energy requirement was given. All rats were allowed to drink water freely. After nutrition programme was completed, all rats were weighted and anaesthetised. A middle abdominal incision was made, the abdominal cavity opened, and the inferior vena cava exposed. EDTA-anti-coagulated venous blood samples were collected in sterile vacuum collection tubes via inferior vena cava puncture for Ig quantification, lymphocyte count, flow

Table 1. Formulas of enteral nutrition solution

\begin{tabular}{lc}
\hline Component & Enteral nutrition solution \\
\hline Energy (kcal) & 1500 \\
Energy (kJ) & 6279 \\
Protein $(\mathrm{g} / \mathrm{l})$ & 60 \\
$\mathrm{~N}(\mathrm{~g} / \mathrm{l})$ & $9 \cdot 4$ \\
$\mathrm{NPC}: \mathrm{N}$ & $133: 1$ \\
Fat $(\mathrm{g} / \mathrm{l})$ & $58 \cdot 4$ \\
Carbohydrate $(\mathrm{g} / \mathrm{l})$ & 185 \\
Composition of total energy $(\%)$ & \\
$\quad$ Protein & 16 \\
Fat & 35 \\
Carbohydrate & 49 \\
\hline
\end{tabular}

NPC, non-protein calories. 
cytometric analysis of $\mathrm{T}$ lymphocyte subsets, plasma GLN concentration and plasma cytokines assay, and the evaluation of neutrophil phagocytosis function. In addition, thymus, spleen and skeletal muscle were harvested for the analysis of GLN concentration, organ index and/or terminal deoxynucleotidyl transferase dUTP nick end labelling (TUNEL).

\section{Ig quantification in serum}

Serum IgA, IgM and IgG contents were quantified by ELISA. In brief, ninety-six-well polystyrene plates were coated with anti-rat IgA, IgM or IgG monoclonal antibodies $(2 \mathrm{mg} / \mathrm{ml}$ in PBS; BD Biosciences) and, after blocking with $1 \%$ bovine serum albumin (Sigma-Aldrich) in PBS ( $1 \mathrm{~h}$, room temperature), appropriate diluted samples and standard dilutions (purified IgA, IgM or IgG; BD Biosciences) were added. After washing, biotin-conjugated anti-rat IgA, IgM or IgG monoclonal antibodies (BD Biosciences) and subsequently peroxidase-conjugated ExtrAvidin (Sigma-Aldrich) were added, respectively. Ano-phenylenediamine dihydrochloride$\mathrm{H}_{2} \mathrm{O}_{2}$ solution (Sigma-Aldrich) was used for the detection of bound peroxidase. $3 \mathrm{M}-\mathrm{H}_{2} \mathrm{SO}_{4}$ was added to stop the reaction. Optical density was measured on a microtitre microplate photometer (Labsystems Multiskan) at $492 \mathrm{~nm}$. Data were interpolated by means of Ascent version 2.6 software (Thermo Fisher Scientific) into the standard curves, and expressed as $\mu \mathrm{g} / \mathrm{ml}$ in serum.

\section{Lymphocyte count in circulation}

Lymphocyte count in circulation was determined by Coulter LH-750 Analyzer (A Beckman Coulter Company).

\section{T lymphocyte subsets in circulation}

The proportions of CD3, CD 4 and CD8 T lymphocyte subsets in circulating blood were measured by flow cytometry. In brief, $100 \mu \mathrm{l}$ of anti-coagulated blood were incubated with either isotypic control or monoclonal antibodies against fluorescein isothiocyanate-CD3, or allophycocyanin-CD4, or peridininchlorophyll proteins-CD8 (BD Pharmingen ${ }^{\mathrm{TM}}$ ) for $30 \mathrm{~min}$ at room temperature in the dark. After haemolysin was added, stained cells were washed twice with cold PBS and assayed by flow cytometry (Becton-Dickinson, Inc.).

\section{The glutamine concentration in plasma, thymus, spleen and skeletal muscle}

The GLN concentration in plasma, thymus, spleen and skeletal muscle was determined by reversed-phase HPLC (Shimadzu LC-6A). The samples were prepared as described previously ${ }^{(29)}$. GLN was pre-column derivatised with o-phthalaldehyde and 3-mercaptopropionic acid under an alkaline condition and separated by HPLC on a LiChrosorb C18 column (Shimadzu). The mobile phase was a mixture of $50 \mathrm{mmol} / 1$ phosphate buffer ( $\mathrm{pH} 7 \cdot 0)$ and acetonitrile $(94: 6, \mathrm{v} / \mathrm{v})$. The flow rate was $2.0 \mathrm{ml} / \mathrm{min}$. A volume of $40 \mu \mathrm{l}$ of the sample was vortex-mixed with $10 \mu$ l derivatisation reagent solution for $2 \mathrm{~min}$. Then, a volume of $20 \mu \mathrm{l}$ of the reaction mixture was injected into the HPLC system. The excited and the emitted wavelengths were selected at 230 and $389 \mathrm{~nm}$, respectively. The result was expressed as $\mu \mathrm{mol} / 1$ plasma or $\mu \mathrm{mol} / \mathrm{g}$ tissues.

\section{The indexes of thymus and spleen}

Thymus and spleen were harvested and weighted. The index of thymus was obtained by thymus net weight divided by the rat body weight, likewise does the index of spleen. The results were expressed as mg (thymus or spleen net weight)/g (rat body weight).

\section{Terminal deoxynucleotidyl transferase dUTP nick end labelling staining of thymus and spleen}

Thymus and spleen were collected and fixed in 10\% neutral buffered formalin and embedded in paraffin. Sections of $4 \mu \mathrm{m}$ thickness were affixed to slides, deparaffinised, rehydrated in graded alcohol (100, 95 and 85\%) and stained with haematoxylin and eosin to evaluate morphological changes. For identification of apoptotic cells, TUNEL staining was conducted by commercially available kits (Wuhan Boster Biological Technology, Limited). All steps were performed according to the supplier's instructions. In brief, paraffin-embedded sections were dewaxed, rehydrated and then incubated with proteinase $\mathrm{K}(20 \mu \mathrm{g} / \mathrm{ml}$ in $100 \mathrm{~mm}$-Tris and $50 \mathrm{~mm}$-EDTA) for $15 \mathrm{~min}$ at $25^{\circ} \mathrm{C}$ to digest proteins, and endogenous peroxidase activity was quenched with $2 \%$ $\mathrm{H}_{2} \mathrm{O}_{2}$ in PBS. After the slides were washed four times with distilled water, the sections were incubated in equilibration buffer for $5 \mathrm{~min}$. The sections were then incubated with the labelling solution containing terminal deoxynucleotidyl transferase in a humidified chamber for $1 \mathrm{~h}$ at $37^{\circ} \mathrm{C}$. The reactions were terminated by rinsing the sections in a stop/wash buffer. Next, the sections were incubated with anti-digoxygenin peroxidase for $30 \mathrm{~min}$ at room temperature. Sections were again washed, and diaminobenzidine $-\mathrm{H}_{2} \mathrm{O}_{2}$ was used for colour development. Sections were then counterstained with $2 \%$ haematoxylin and mounted for examination.

TUNEL analysis was conducted according to the method described previously ${ }^{(30)}$. Lymphocytes were identified by morphology, with cell sizes of approximately $10 \mu \mathrm{m}$ and low cytoplasm:nucleus ratios. Apoptotic cells were identified either as cells with brown-stained nuclei or as apoptotic bodies, which are fragments of apoptotic cells engulfed by neighbouring cells. The number of TUNEL-positive nuclei is enumerated for two random high power fields per tissue section by three reviewers blinded as to the sample origin. Averaging the number of TUNEL-positive nuclei from each field for all three reviewers resulted in an overall TUNEL-positive score for the tissue. The results were expressed as TUNEL-staining cells/ high power fields.

\section{Plasma cytokine assay}

To clarify the change of inflammatory cytokine, the levels of TNF- $\alpha$, IL-1 $\beta$, IL-6 and IL-10 in plasma were analysed by 
ELISA. Briefly, the plasma was separated by centrifugation at $16000 \mathrm{~g}$ for $15 \mathrm{~min}$ at $4^{\circ} \mathrm{C}$, aliquoted, immediately frozen and stored $\left(-84^{\circ} \mathrm{C}\right)$ until assayed. The levels of TNF- $\alpha$, IL-1 $\beta$, IL-6 and IL-10 were determined by commercially available ELISA kits (R\&D Systems) according to the manufacturer's recommendations. The results were expressed as $\mathrm{pg} / \mathrm{ml}$ or $\mathrm{ng} / \mathrm{ml}$.

\section{Neutrophil phagocytosis index}

Neutrophil phagocytosis index (NPI) was determined according to the method as described previously ${ }^{(25)}$. Briefly, about $2 \mathrm{ml}$ of anti-coagulated blood was collected in a test-tube containing $0.4 \mathrm{ml}$ of $6 \%$ dextran $\mathrm{T} 500$ (Pharmacia). The test-tube was placed upright for $45-60 \mathrm{~min}$ to allow the separation of red cells and plasma. The plasma layer was then removed to centrifuge tube, centrifuged at $2000 \boldsymbol{g}$ for $5 \mathrm{~min}$. After centrifugation, the mononuclear layer was removed to another centrifuge tube. Neutrophils contaminating erythrocytes was resuspended. The erythrocytes were removed by hypotonic lysis. The cells were washed two times with Hanks' balanced salt solution and suspended in $1 \times$ Hanks' balanced salt solution. Finally, cellular viability was evaluated using the trypan blue exclusion technique. The purity and viability of neutrophils for each assay were $>97 \%$. The neutrophils were counted and adjusted to a concentration of $1 \times 10^{6}$ cells/ml.

Neutrophils $\left(5 \times 10^{6}\right)$ were resuspended in Roswell Park Memorial Institute-1640 medium (Gibco) containing 10\% (v/v) fetal bovine serum (Gibco) and subsequently incubated with $1 \times 10^{7}$ Staphylococcus aureus in a final volume of $1 \mathrm{ml}$ for $2 \mathrm{~h}$ at $37^{\circ} \mathrm{C}$. The NPI was determined by microscopic observation after stained with Gram's stain. Under a light microscope, NPI $=$ cells of phagocytosis bacteria/total cells $\times 100 \%$.

\section{Survival studies}

A separate set of forty male Sprague-Dawley rats was served as survival study. CLP or sham CLP model, grouping and nutrition programme were the same as described above. When nutrition programme began and went on, animals were observed at regular intervals for occurrence of mortality for $7 \mathrm{~d}$.

\section{Statistical analysis}

Data were expressed as the means with standard deviations, and differences among groups were analysed by ANOVA using Newman-Keuls' test. Fisher's exact test was utilised to compare survival data. Values of $P<0.05$ were regarded as significant.

\section{Results}

\section{Body weight change}

As shown in Table 2, it was not observed that there was obvious difference in body weight at the beginning of the experiment among the four groups $(P>0.05)$. However, at the end of the experiment, the rats lost more body weight in two experimental groups (the CLP+EN+AA group and the CLP+EN+GLN group) than that in two sham CLP control groups (the sham CLP+EN+AA group and the sham CLP+ EN+GLN group; $P<0.05)$. There was no difference in body weight between the CLP+EN+AA group and the CLP+EN+ GLN group $(P>0 \cdot 05)$.

\section{Neutrophil phagocytosis index}

As shown in Table 2, NPI from the CLP rats were decreased when compared with the sham CLP rats $(P<0 \cdot 05)$. However, NPI from the CLP+EN+GLN group were significantly increased compared with the CLP+EN+AA group $(P<0 \cdot 05)$.

\section{Ig quantification in serum}

As shown in Table 3, the contents of IgA, IgG and IgM in serum in two experimental groups were significantly decreased compared with two sham CLP control groups $(P<0.05)$. However, the contents of IgA, IgG and IgM in the $\mathrm{CLP}+\mathrm{EN}+\mathrm{GLN}$ group were higher than those in the CLP+ EN+AA group $(P<0 \cdot 05)$.

\section{Number of lymphocyte in circulation}

As shown in Table 3, the numbers of circulating lymphocyte in two experimental groups were apparently small compared with two sham CLP control groups $(P<0.05)$. However, the

Table 2. The changes of body weight and neutrophil phagocytosis index (NPI) (Mean values and standard deviations)

\begin{tabular}{|c|c|c|c|c|c|c|c|}
\hline \multirow[b]{3}{*}{ Group } & \multirow[b]{3}{*}{$n$} & \multicolumn{4}{|c|}{ Change of body weight } & & \\
\hline & & \multicolumn{2}{|c|}{$\begin{array}{l}\text { Body weight before } \\
\text { experiment }(\mathrm{g})\end{array}$} & \multicolumn{2}{|c|}{$\begin{array}{l}\text { Loss of body weight } \\
\text { after experiment }(\mathrm{g})\end{array}$} & \multicolumn{2}{|c|}{ NPI (\%) } \\
\hline & & Mean & SD & Mean & SD & Mean & SD \\
\hline Sham CLP+EN+AA & 10 & $242 \cdot 3$ & $24 \cdot 2$ & $-10 \cdot 2$ & 0.8 & 43.8 & $4 \cdot 2$ \\
\hline Sham CLP+EN+GLN & 10 & $238 \cdot 2$ & $26 \cdot 4$ & -11.5 & 1.0 & $45 \cdot 2$ & $4 \cdot 3$ \\
\hline $\mathrm{CLP}+\mathrm{EN}+\mathrm{AA}$ & 10 & 238.6 & $24 \cdot 6$ & $-35 \cdot 4^{\star}$ & $2 \cdot 8$ & $24 \cdot 7^{\star}$ & $2 \cdot 6$ \\
\hline $\mathrm{CLP}+\mathrm{EN}+\mathrm{GLN}$ & 10 & $240 \cdot 5$ & 24.5 & $-36 \cdot 3^{*}$ & 2.4 & $33 \cdot 6^{*} \dagger$ & 3.5 \\
\hline
\end{tabular}

CLP, caecal ligation and puncture; EN, enteral nutrition; AA, glutamine-free amino acid; GLN, glutamine.

${ }^{*}$ Mean value was significantly different from that of the sham CLP+EN+AA group or the sham $\mathrm{CLP}+\mathrm{EN}+\mathrm{GLN}$ group $(P<0.05)$.

† Mean value was significantly different from that of the CLP+EN+AA group $(P<0.05)$. 
number of circulating lymphocyte in the CLP+EN+GLN group was bigger than that in the CLP+EN+AA group $(P<0 \cdot 05)$.

As shown in Fig. 1 and Table 3, CLP led to changes in the percentage of $\mathrm{T}$ lymphocyte subsets in circulation in the CLP rats. The percentage of $\mathrm{CD} 4$ cells was remarkably decreased, whereas that of CD8 cells was correspondingly increased $(P<0.05)$, which led to decreased CD4:CD8 ratio $(P<0 \cdot 05)$. EN combined with parenteral GLN supplementation partially reversed the changes and the percentages of CD4 and CD8 cells and the CD4:CD8 ratio returned to the sham CLP levels $(P>0.05)$.

\section{Glutamine concentrations in plasma, thymus, spleen and skeletal muscle}

As shown in Table 4, the GLN concentrations of plasma, thymus, spleen and skeletal muscle in two experimental groups were apparently decreased compared with the two sham CLP control groups $(P<0.05)$. However, there was a notable increment in the GLN concentrations of plasma, thymus, spleen and skeletal muscle in the CLP+EN+GLN group compared with the CLP+EN+AA group $(P<0 \cdot 05)$.

\section{Cytokines in plasma}

As shown in Table 4, levels of TNF- $\alpha$, IL-1 $1 \beta$, IL- 6 and IL-10 in plasma from the CLP rats were increased when compared with the sham CLP rats $(P<0 \cdot 05)$. The present results revealed that CLP markedly increased the release of pro- and anti-inflammatory mediators. However, levels of TNF- $\alpha$, IL- $1 \beta$ and IL- 6 in plasma in the CLP+EN+GLN group were significantly decreased compared with the CLP+EN+AA group $(P<0 \cdot 05)$. There was no obvious difference in level of IL-10 between the CLP+EN+GLN group and the CLP+EN+AA group $(P>0.05)$.

\section{The indexes of thymus and spleen}

As shown in Table 5, CLP caused a significant atrophy in the size and weight of thymus and spleen, which was perfectly consistent with evidently decreased indexes of thymus and spleen. However, the indexes of thymus and spleen were markedly enlarged in the CLP+EN+GLN group compared with the CLP+EN+AA group $(P<0 \cdot 05)$.

\section{Terminal deoxynucleotidyl transferase dUTP nick end labelling analysis of thymus and spleen apoptosis}

To determine whether the observed increase in the indexes of thymus and spleen conferred by EN combined with parenteral GLN supplementation was associated with a reduction in thymus and spleen apoptotic death, apoptosis in thymus and spleen was analysed by TUNEL staining. As shown in Figs. 2, 3 and Table 5, TUNEL-staining cells/high power fields in thymus and spleen from CLP rats were dramatically increased when compared with the sham CLP rats $(P<0 \cdot 05)$. Yet, TUNEL-staining cells/high power fields in thymus and 
(a)

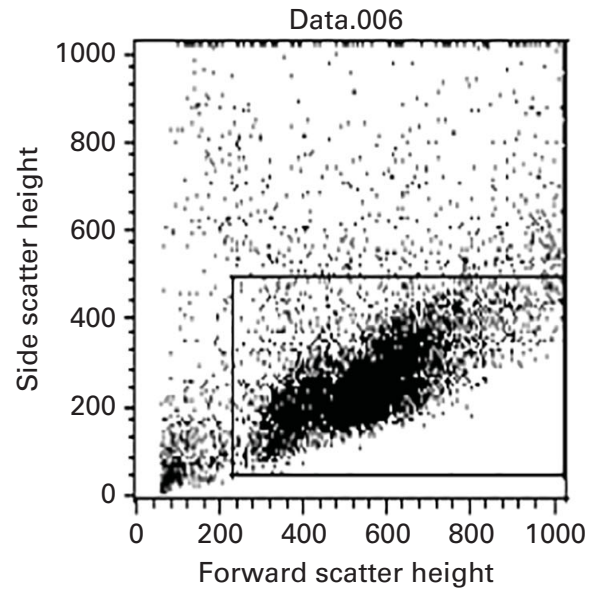

(c)

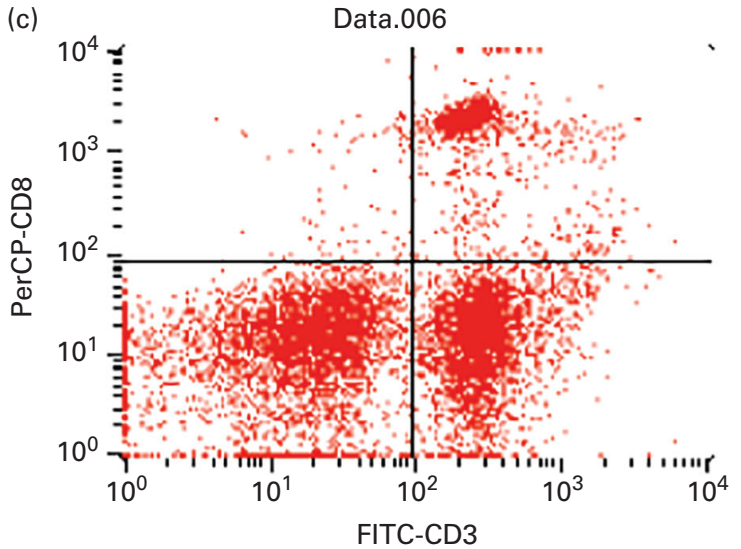

(b)

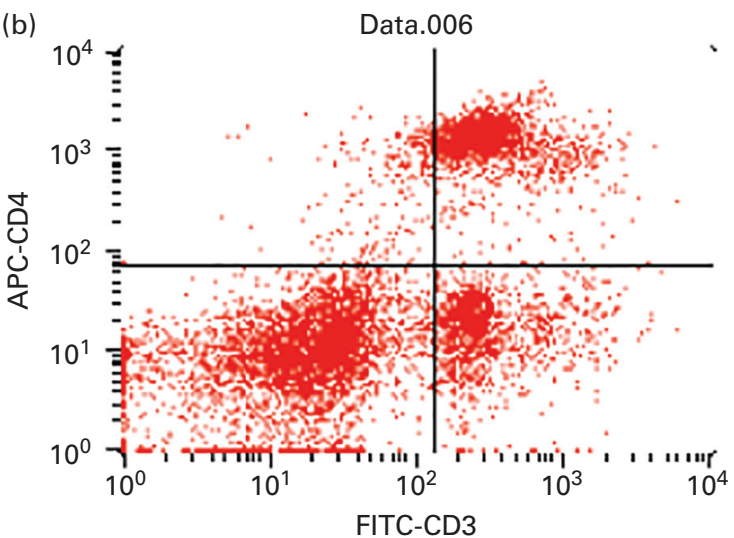

(d)

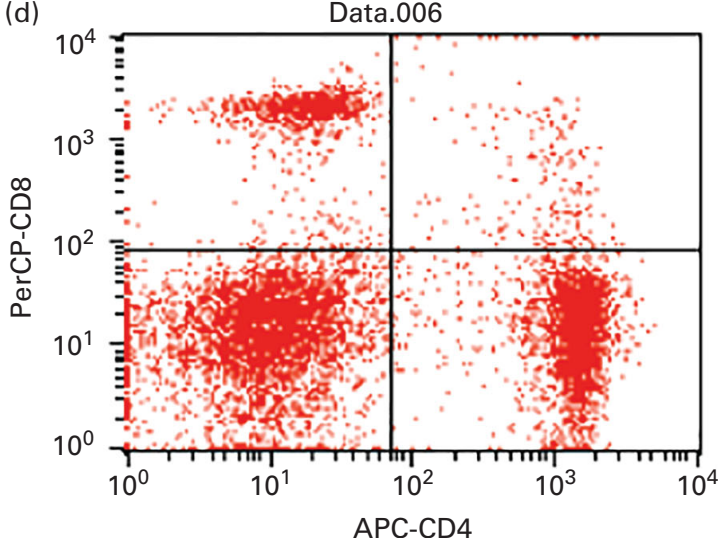

Fig. 1. Flow cytometry dot plot analyses of $T$ lymphocyte subsets in circulating blood. (a) Whole lymphocyte gate; (b) fluorescein isothiocyanate (FITC)-conjugated mouse anti-rat $\mathrm{CD} 3$ and allophycocyanin (APC)-conjugated mouse anti-rat $\mathrm{CD} 4$ to determine $\mathrm{CD} 3^{+} \mathrm{CD} 4^{+}$double-positive T lymphocyte; (c) FITC-conjugated mouse anti-rat CD3 and peridinin-chlorophyll proteins (PerCP)-conjugated mouse anti-rat CD8 to determine $\mathrm{CD} 3^{+} \mathrm{CD} 8^{+}$double-positive $\mathrm{T}$ lymphocyte and (d) the $\mathrm{CD} 4: \mathrm{CD} 8$ ratio. A colour version of this figure can be found online at http://www.journals.cambridge.org/bjn

spleen in the CLP+EN+GLN group had a marked decrease compared with the CLP+EN+AA group $(P<0.05)$.

\section{Survival rate}

Animals were observed for $7 \mathrm{~d}$ for survival rate. No mortality occurred in the two sham CLP control groups. The survival rate in the CLP+EN+GLN group was $60 \%$, whereas that in the CLP+EN+AA group was $40 \%$. The survival rate of the CLP rats was significantly decreased in comparison to the sham CLP rats $(P<0.05)$. Nevertheless, EN with parenteral GLN supplementation improved survival of the CLP rats. The survival rate in the CLP+EN+GLN group was significantly increased when compared with the CLP+EN+AA group $(P<0 \cdot 05)$.

\section{Discussion}

The CLP model of sepsis in rodent is the most commonly used for studying the process of septic peritonitis and can be used as a preclinical model to test the efficacy of pharmacological agents for the treatment of sepsis ${ }^{(31)}$. We attempted to explore whether EN with parenteral GLN supplementation could improve humoral and cellular immunity as well as modulate the imbalance of inflammatory response by a CLP rat model.

With respect to the dosage of GLN, the dosage of $0.35 \mathrm{~g} / \mathrm{kg}$ per $d$ GLN was chosen in accordance with the clinical relevance and the results of our preliminary experiment. Some studies have reported that there is a trend to an increased mortality in those patients treated with a high dose of GLN ( $1 \mathrm{~g} / \mathrm{kg}$ per $\mathrm{d} \mathrm{GLN})^{(32)}$. In our preliminary experiment, we found that the dosage of $0.35 \mathrm{~g} / \mathrm{kg}$ body weight per $\mathrm{d}$ GLN was safe, effect and appropriate, and had good clinical relevance.

The most important finding in the present study confirmed that parenteral GLN supplementation could effectively attenuate thymus and spleen apoptosis, subsequently boosting cellular and humoral immunity in septic rats.

In the present study, we observed that CLP led to an obvious decrease in plasma Ig and circulating lymphocyte count. Meanwhile, the percentage of CD4 cells was declined and that of CD8 cells was increased, which resulted in an obviously decreased CD4:CD8 ratio. Additionally, NPI significantly decreased. Neutrophils are critical components of the innate immune response to infectious challenges ${ }^{(4)}$. The present results confirmed that humoral- and cellular immune 
function was suppressed after CLP. Clinical data also documented that there was a significant decrease in circulating lymphocytes from septic patients ${ }^{(33-36)}$. Other data have reported that phagocytic and bactericidal function of neutrophils was impaired after major operation, severe burn and sepsis ${ }^{(25,37,38)}$. The principal outcome of such immune suppression is the increased susceptibility of injured host to opportunistic pathogens causing high risk of death ${ }^{(34)}$. However, this negative situation might be partially reversed by EN combined with intravenous GLN supplementation. The present study disclosed that there was an apparent amelioration in humoral immune function such as Ig content and cellular immune function including lymphocyte count, the CD4:CD8 ratio and NPI in the CLP+EN+GLN group, which would be beneficial to the improvement of antiinfectious immune function.

The present study also measured the changes of the GLN concentrations of plasma, thymus, spleen and skeletal muscle by HPLC in the CLP rats. The results showed that the stress, injury and infection owing to CLP contributed to a rapid decrease in the GLN concentrations of plasma, thymus, spleen and skeletal muscle. Lymphocyte is very sensitive to alterations in nutritional substrate supply, especially in GLN supply ${ }^{(39)}$. Indeed, rapidly dividing immune cells deeply depend on the availability of GLN as an energy (C and N) source $^{(25)}$. Also, GLN functions as a $\mathrm{N}$ shuttle, carrying $\mathrm{N}$ for purine and pyrimidine synthesis, which are essential for DNA and RNA synthesis ${ }^{(9)}$. Further, immune cells require more GLN during inflammation and stress in which cell proliferation is dramatically increased ${ }^{(25)}$. Sepsis frequently causes hypercatabolic response ${ }^{(40)}$, which is characterised by a significant increase in energy requirements, BMR, tissue $\mathrm{V}_{\mathrm{O} 2}$, $\mathrm{N}$ and body weight loss ${ }^{(41)}$. Because of the impairment of the ability to use glucose and fat, there is an increased dependency on muscle breakdown, providing essential substrates for acute phase protein synthesis and energy production $^{(42)}$. Skeletal muscle, as the major repository of GLN, can obviously increase GLN release during stress and severe infection ${ }^{(43)}$. Despite an increase in GLN release from skeletal muscle, the intracellular GLN pool becomes depleted, indicating that release rates exceed rates of synthesis. Simultaneously, the circulating pool of GLN does not increase, indicating accelerated uptake by other organs such as intestine, liver and the immune system ${ }^{(43)}$. GLN requirements increase markedly, and its consumption in visceral organs frequently exceeds its release from muscle ${ }^{(44)}$. Thus, it may cause protein-energy malnutrition and specific nutrient deficiency such as GLN. GLN depletion decreases proliferation of lymphocytes, affects expression of surface activation markers on lymphocytes and the production of cytokines and induces apoptosis ${ }^{(45)}$. GLN supplementation may partially reverse the changes. The present results displayed that EN combined with intravenous GLN supplementation remarkably increased GLN concentrations of plasma, thymus, spleen and skeletal muscle in the CLP rats, which improved GLN metabolism and exerted beneficial effects on the improvement of immunological function during the inflammation and stress. 
Table 5. The changes of organ index and apoptosis in thymus and spleen

(Mean values and standard deviations)

\begin{tabular}{|c|c|c|c|c|c|c|c|c|c|}
\hline \multirow[b]{3}{*}{ Group } & \multirow[b]{3}{*}{$n$} & \multicolumn{4}{|c|}{ Thymus } & \multicolumn{4}{|c|}{ Spleen } \\
\hline & & \multicolumn{2}{|c|}{$\begin{array}{l}\text { Organ index } \\
\qquad(\mathrm{mg} / \mathrm{g})\end{array}$} & \multicolumn{2}{|c|}{$\begin{array}{l}\text { TUNEL-staining } \\
\text { cells/hpf }\end{array}$} & \multicolumn{2}{|c|}{$\begin{array}{l}\text { Organ index } \\
(\mathrm{mg} / \mathrm{g})\end{array}$} & \multicolumn{2}{|c|}{$\begin{array}{l}\text { TUNEL-staining } \\
\text { cells/hpf }\end{array}$} \\
\hline & & Mean & SD & Mean & $\mathrm{SD}$ & Mean & SD & Mean & SD \\
\hline Sham CLP+EN+AA & 10 & $2 \cdot 1$ & 0.2 & 23 & 2 & $2 \cdot 6$ & 0.2 & 18 & 1 \\
\hline Sham CLP+EN+GLN & 10 & $2 \cdot 2$ & 0.2 & 25 & 2 & 2.5 & 0.2 & 16 & 1 \\
\hline $\mathrm{CLP}+\mathrm{EN}+\mathrm{AA}$ & 10 & $0.9^{*}$ & 0.1 & $136^{*}$ & 9 & $1 \cdot 2^{*}$ & 0.1 & $82^{*}$ & 7 \\
\hline $\mathrm{CLP}+\mathrm{EN}+\mathrm{GLN}$ & 10 & $1.4^{*} \dagger$ & $0 \cdot 1$ & $62^{*} \dagger$ & 4 & $2 \cdot 0^{\star} \dagger$ & 0.1 & $43^{*} \dagger$ & 4 \\
\hline
\end{tabular}

TUNEL, terminal deoxynucleotidyl transferase dUTP nick end labelling; hpf, high power fields; CLP, caecal ligation and puncture; EN, enteral nutrition; AA, glutamine-free amino acid; GLN, glutamine.

* Mean value was significantly different from that of the sham CLP+EN+AA group or the sham CLP+EN+GLN group $(P<0.05)$.

$\dagger$ Mean value was significantly different from that of the CLP+EN+AA group $(P<0.05)$.

In the present study, quantified analysis of TUNEL staining disclosed that apoptosis in thymus and spleen from the CLP rats was dramatically increased, which was closely correlated with an obvious atrophy in the size and weight of thymus and spleen, and decreased circulating lymphocytes. In fact, appropriate lymphocyte apoptosis may be beneficial to septic patients by down-regulating the excessive inflammatory response ${ }^{(46)}$. However, exaggerated apoptotic cell death may decrease immune cells available to combat pathogens. It is proposed that in the condition of stress and severe injury, apoptosis-induced extensive loss of lymphocytes in the central and peripheral immune system may lead to immunocompromising, leaving the patients vulnerable to subsequent infections or unable to mount an appropriate host response to invading pathogens ${ }^{(47)}$. Extensive experimental and clinical data support the hypothesis that one of the major pathophysiological mechanisms in sepsis is uncontrolled, extensive and accelerated lymphocyte apoptosis ${ }^{(33,34,46-48)}$. Therefore, modulating the imbalance of apoptosis response of immune cell might present another attractive therapy target for sepsis ${ }^{(46-48)}$. The present study indicated that EN with parenteral GLN supplementation decreased apoptosis in thymus and spleen. Other studies have reported that GLN supplementation could attenuate
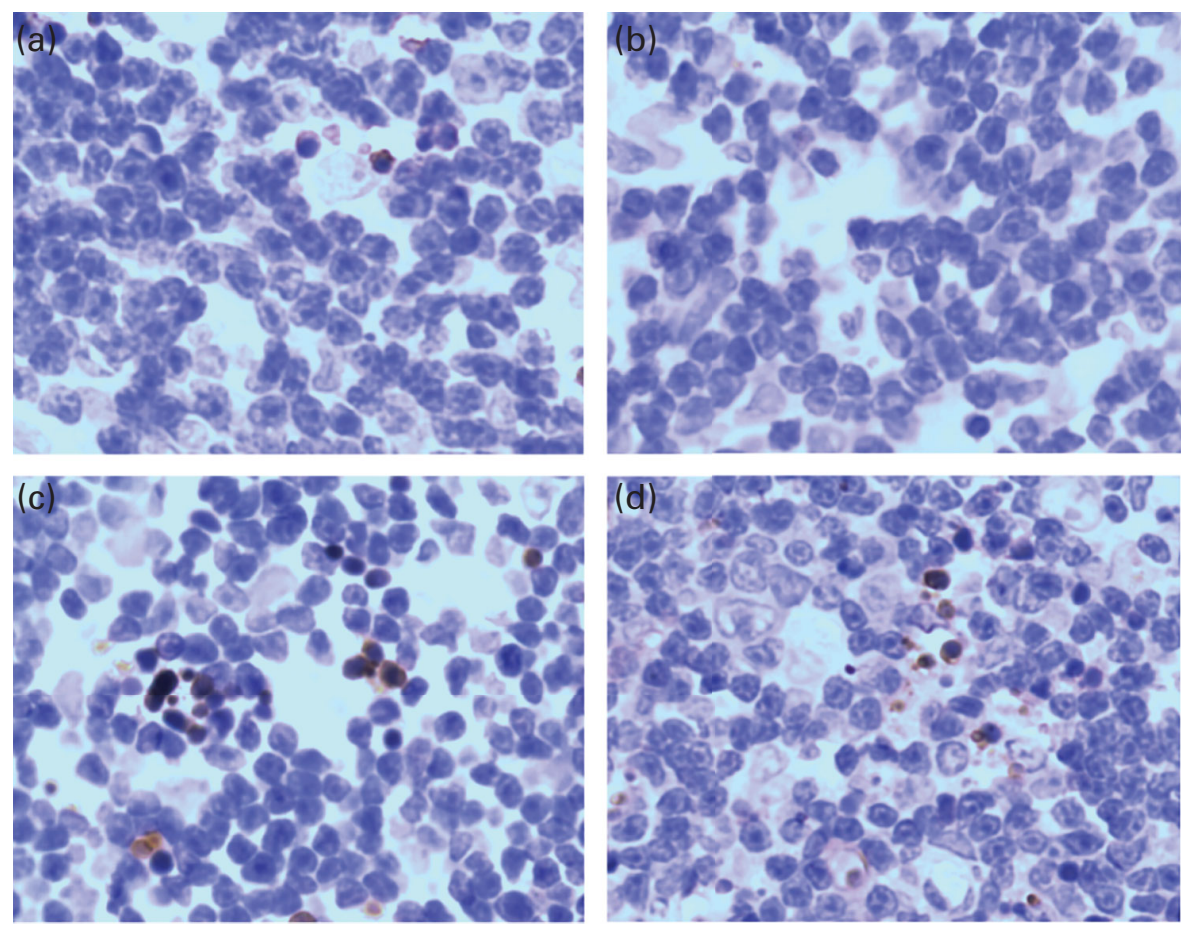

Fig. 2. Terminal deoxynucleotidyl transferase dUTP nick end labelling (TUNEL) analysis of thymus lymphocyte apoptosis from rats. (a-d) Representative photomicrographs of TUNEL-stained thymus sections in the sham caecal ligation and puncture (CLP)+enteral nutrition (EN) + glutamine (GLN)-free amino acid (AA), sham $C L P+E N+G L N, C L P+E N+A A$ and $C L P+E N+G L N$ group, respectively. (a, b) Sporadic apoptotic lymphocytes without apparent clustering of apoptotic cells are scattered throughout thymus in a random distribution. (c) Apparent clustering of apoptotic cells are scattered throughout thymus in a random distribution in the CLP+EN+AA group. (d) Apparently decreased apoptotic cells are scattered throughout thymus in a random distribution in the CLP+EN+GLN group. TUNEL-positive (apoptotic) nuclei appear in crown $\times 400$. A colour version of this figure can be found online at http://www.journals.cambridge.org/bjn 

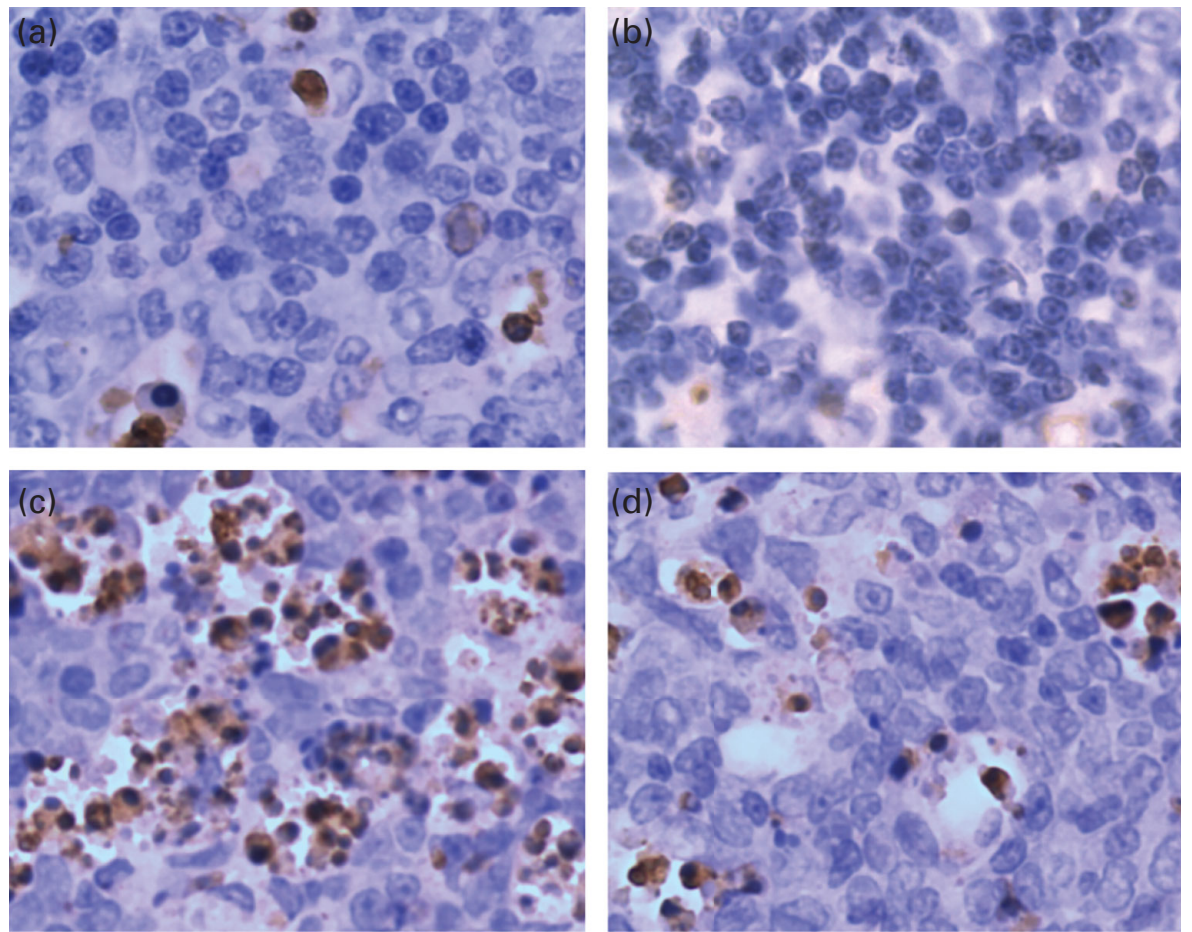

Fig. 3. Terminal deoxynucleotidyl transferase dUTP nick end labelling (TUNEL) analysis of spleen lymphocyte apoptosis from rats. (a-d) Representative photomicrographs of TUNEL-stained spleen sections in the sham caecal ligation and puncture (CLP)+enteral nutrition (EN)+glutamine (GLN)-free amino acid (AA), sham CLP+EN+GLN, CLP+EN+AA and CLP+EN+GLN groups, respectively. (a, b) Sporadic apoptotic lymphocytes without apparent clustering of apoptotic cells are scattered throughout spleen in a random distribution. (c) Apparent clustering of apoptotic cells are scattered throughout spleen in a random distribution in the CLP+EN+AA group. (d) Apparently decreased apoptotic cells are scattered throughout spleen in a random distribution in the CLP+EN+GLN group. TUNELpositive (apoptotic) nuclei appear in crown $\times 400$. A colour version of this figure can be found online at http://www.journals.cambridge.org/bjn

apoptosis occurring in heart, lung, liver, kidney and intestine during severe stress and inflammation ${ }^{(49-53)}$. For instance, Yin et $a l{ }^{(49)}$ demonstrated that GLN reduced myocardial apoptotic cell death by decreasing the gene and protein expression of caspase-3. Also, de Oliveira et al. ${ }^{(50)}$ certified that early therapy with intravenous GLN reduced inflammation, fibrosis and apoptosis, minimising lung and distal organ injury. Moreover, Hu et al. ${ }^{(21)}$ found that GLN prevented apoptosis of $\mathrm{T}$ cells, which may partly be responsible for ameliorating acute lung injury induced by sepsis. Kim et al. ${ }^{(51)}$ showed that GLN attenuated tubular cell apoptosis in acute kidney injury via inhibition of the c-Jun N-terminal kinase phosphorylation of $14-3-3$. In addition, Uehara et al. ${ }^{(52)}$ proved that GLN pretreatment significantly ameliorated intestinal injury by preventing apoptosis. Furthermore, Margaritis et $a l{ }^{(53)}$ documented that GLN in the presence of obstructive jaundice ameliorated bacterial tanslocation, endotoxemia and apoptosis and improved the ileal and liver histology. Therefore, it is possible that GLN becomes a clinically relevant nutrient that modulates the imbalance of apoptotic response.

The present study also evaluated the impact of EN with parenteral GLN supplementation on the levels of TNF- $\alpha$, IL-1 $\beta$, IL- 6 and IL-10 in plasma in the CLP rats. The results revealed that the levels of TNF- $\alpha$, IL- $1 \beta$, IL- 6 and IL-10 in plasma from the CLP rats markedly increased, and that EN with intravenous GLN supplementation obviously decreased the levels of TNF- $\alpha$, IL- $1 \beta$ and IL- 6 in plasma. Other investigations also supported that GLN could attenuate pro-inflammatory response in animal and clinical experiments ${ }^{(11-18)}$. Some studies have also explored possible mechanisms by which GLN decrease the release of pro-inflammatory cytokines. For instance, Kessel et al. ${ }^{(54)}$ found that GLN could effectively down-regulate the expression of Toll-like receptor 4, myeloid differentiation factor 88 and $\mathrm{TNF}-\alpha$ receptor-associated factor 6 , concomitantly decreasing intestinal injury in endotoxemia rats. In addition, Singleton et al. ${ }^{(55)}$ confirmed that GLN could inhibit the activation of the stress kinase pathway, including p38 mitogen-activated protein kinase and extracellular signal-regulated kinase, which led to decreased TNF- $\alpha$ and IL-6 expression after sepsis. As cytokine levels may change drastically over time in sepsis, their measurement at only one time point should be acknowledged as limitations in the present study.

In the present study, we also observed the influence of EN with parenteral GLN supplementation on survival in the CLP rats. The result showed that CLP resulted in significantly increased mortality in the CLP rats, that EN with parenteral GLN supplementation significantly improved survival in the CLP rats, which was consistent with another investigation ${ }^{(56)}$. It is probably for the reasons that parenteral GLN supplementation increases the GLN concentrations in plasma and tissues, attenuates immune organ apoptosis, enhances humoral- and cellular immunity and down-regulates the levels of TNF- $\alpha$, IL-1 $\beta$ and IL- 6 in plasma responsible for the change.

In conclusion, the present findings show that EN with parenteral GLN supplementation may decrease the release of 
pro-inflammatory cytokines, attenuate lymphatic organ apoptosis, enhance immune function and improve survival in septic rats. Clinically, these results suggest that immunomodulating therapy by GLN supplementation may affect favourably on outcome of septic patients. In addition, more research is needed to explore the mechanisms by which GLN may prevent apoptosis. They would be further studied in the future research.

\section{Acknowledgements}

The present study was supported by the National Natural Science Foundation of China (no. 81160240), China Postdoctoral Science Foundation (no. 20080440231), Science and Technology Support Foundation of Jiangxi Provincial Department of Science and Technology (no. 2010BSB00203), Science Foundation of Department of Health of Jiangxi Province (no. 20121059).

The authors' contributions are as follows: J. F. involved in the study concept and design; J. F., L. W., G. L., S. T., Z. S., F. L., L. Y. and L. L. involved in the performance of the experiment; J. F. and Q. M. involved in the analysis and interpretation of the data; J. F. involved in the responsibility for the integrity of the data and the accuracy of the data analysis; J. F. involved in the preparation of the manuscript.

The authors declare that they have no conflict of interest.

\section{References}

1. Boomer JS, Green JM \& Hotchkiss RS (2014) The changing immune system in sepsis: is individualized immunomodulatory therapy the answer? Virulence 5, 45-56.

2. Muenzer JT, Davis CG \& Chang K (2010) Characterization and modulation of the immunosuppressive phase of sepsis. Infect Immun 78, 1582-1592.

3. Hutchins NA, Unsinger J \& Hotchkiss RS (2014) The new normal: immunomodulatory agents against sepsis immune suppression. Trends Mol Med 20, 224-233.

4. Remick DG (2007) Pathophysiology of sepsis. Am J Pathol 170, 1435-1444.

5. Santora R \& Kozar RA (2010) Molecular mechanisms of pharmaconutrients. J Surg Res 161, 288-294.

6. Anbar R (2013) Enteral nutrition. World Rev Nutr Diet 105, $50-58$.

7. Elke G, Wang $\mathrm{M}$, Weiler $\mathrm{N}$, et al. (2014) Close to recommended caloric and protein intake by enteral nutrition is associated with better clinical outcome of critically ill septic patients: secondary analysis of a large international nutrition database. Crit Care 18, R29.

8. Braga M, Wischmeyer PE, Drover J, et al. (2013) Clinical evidence for pharmaconutrition in major elective surgery. JPEN J Parenter Enteral Nutr 37, 665-72S.

9. Lacey JM \& Wilmore DW (1990) Is glutamine a conditionally essential amino acid? Nutr Rev 48, 297-309.

10. Cruzat VF, Pantaleão LC, Donato J Jr, et al. (2014) Oral supplementations with free and dipeptide forms of L-glutamine in endotoxemic mice: effects on muscle glutamine-glutathione axis and heat shock proteins. J Nutr Biochem 25, 345-352.

11. Hou YC, Chiu WC, Yeh CL, et al. (2012) Glutamine modulates lipopolysaccharide-induced activation of NF-кB via the Akt/mTOR pathway in lung epithelial cells. Am J Physiol Lung Cell Mol Physiol 302, L174-L183.
12. Lee CH, Kim HK, Jeong JS, et al. (2015) Mechanism of glutamine inhibition of cytosolic phospholipase A2 (cPLA2): evidence of physical interaction between glutamine-induced MAPK phosphatase-1 and cPLA2. Clin Exp Immunol (Epublication ahead of print version).

13. Cruzat VF, Bittencourt A, Scomazzon SP, et al. (2014) Oral free and dipeptide forms of glutamine supplementation attenuate oxidative stress and inflammation induced by endotoxemia. Nutrition 30, 602-611.

14. Hou YC, Pai MH, Liu JJ, et al. (2013) Alanyl-glutamine resolves lipopolysaccharide-induced lung injury in mice by modulating the polarization of regulatory $\mathrm{T}$ cells and $\mathrm{T}$ helper 17 cells. J Nutr Biochem 24, 1555-1563.

15. Kwon WY, Suh GJ, Kim KS, et al. (2010) Glutamine attenuates acute lung injury by inhibition of high mobility group box protein-1 expression during sepsis. BrJ Nutr 103, 890-898.

16. Hu YM, Pai MH, Yeh CL, et al. (2012) Glutamine administration ameliorates sepsis-induced kidney injury by downregulating the high-mobility group box protein-1mediated pathway in mice. Am J Physiol Renal Physiol 302, F150-F158.

17. Zhou W, Li W, Zheng XH, et al. (2014) Glutamine downregulates TLR-2 and TLR-4 expression and protects intestinal tract in preterm neonatal rats with necrotizing enterocolitis. J Pediatr Surg 49, 1057-1063.

18. Joo E, Yamane S, Hamasaki A, et al. (2013) Enteral supplement enriched with glutamine, fiber, and oligosaccharide attenuates experimental colitis in mice. Nutrition 29, 549-555.

19. Fan J, Meng QY, Guo GH, et al. (2009) Effects of enteral nutrition supplemented with glutamine on intestinal mucosal immunity in burned mice. Nutrition 25, 233-239.

20. Fan J, Meng QY, Guo GH, et al. (2010) Effects of glutamine added to enteral nutrition on Peyer's patch apoptosis in severely burned mice. Burns 36, 409-417.

21. Hu YM, Yeh CL, Pai MH, et al. (2014) Glutamine administration modulates lung $\gamma \delta \mathrm{T}$ lymphocyte expression in mice with polymicrobial sepsis. Shock 41, 115-122.

22. Tung JN, Lee WY, Pai MH, et al. (2013) Glutamine modulates $\mathrm{CD} 8 \alpha \alpha(+) \mathrm{TCR} \alpha \beta(+)$ intestinal intraepithelial lymphocyte expression in mice with polymicrobial sepsis. Nutrition 29, 911-917.

23. Lee WY, Hu YM, Ko TL, et al. (2012) Glutamine modulates sepsis-induced changes to intestinal intraepithelial $\gamma \delta \mathrm{T}$ lymphocyte expression in mice. Shock 38, 288-293.

24. Cavalcante AA, Campelo MW, de Vasconcelos MP, et al. (2012) Enteral nutrition supplemented with L-glutamine in patients with systemic inflammatory response syndrome due to pulmonary infection. Nutrition 28, 397-402.

25. Peng X, Yan H, You Z, et al. (2006) Glutamine granulesupplemented enteral nutrition maintains immunological function in severely burned patients. Burns 32, 589-593.

26. Bollhalder L, Pfeil AM, Tomonaga Y, et al. (2013) A systematic literature review and meta-analysis of randomized clinical trials of parenteral glutamine supplementation. Clin Nutr 32, $213-223$

27. Heyland D, Muscedere J, Wischmeyer PE, et al. (2013) A randomized trial of glutamine and antioxidants in critically ill patients. $N$ Engl J Med 368, 1489-1497.

28. Wischmeyer PE, Dhaliwal R, McCall M, et al. (2014) Parenteral glutamine supplementation in critical illness: a systematic review. Crit Care 18, R76.

29. Teerlink T, Hennekes MW, Van Leeuwen PA, et al. (1993) Rapid determination of glutamine in biological samples by high-performance liquid chromatography. Clin Chim Acta 218, 159-168. 
30. Woodside KJ, Spies M, Wu XW, et al. (2003) Decreased lymphocyte apoptosis by anti-tumor necrosis factor antibody in PPs after severe burn. Shock 20, 70-73.

31. Medina E (2010) Murine model of polymicrobial septic peritonitis using cecal ligation and puncture (CLP). Methods Mol Biol 602, 411-415.

32. Andrews PJ, Avenell A \& Noble DW (2011) Randomised trial of glutamine, selenium, or both, to supplement parenteral nutrition for critically ill patients. BMJ 342, d1542.

33. Le Tulzo Y, Pangault C, Gacouin A, et al. (2002) Early circulating lymphocyte apoptosis in human septic shock is associated with poor outcome. Shock 18, 487-494.

34. Hotchkiss RS \& Karl IE (2003) The pathophysiology and treatment of sepsis. $N$ Engl J Med 348, 138-150.

35. Felmet KA, Hall MW, Clark RS, et al. (2005) Prolonged lymphopenia, lymphoid depletion, and hypoprolactinemia in children with nosocomial sepsis and multiple organ failure. J Immunol 174, 3765-3772.

36. Drewry AM, Samra N, Skrupky LP, et al. (2014) Persistent lymphopenia after diagnosis of sepsis predicts mortality. Shock 42, 383-391.

37. Furukawa S, Saito H, Inoue T, et al. (2000) Supplemental glutamine augments phagocytosis and reactive oxygen intermediate production by neutrophils and monocytes from postoperative patients in vitro. Nutrition 16, 323-329.

38. Zou L, Feng Y, Zhang M, et al. (2011) Nonhematopoietic Tolllike receptor 2 contributes to neutrophil and cardiac function impairment during polymicrobial sepsis. Shock 36, 370-380.

39. Manhart N, Vierlinger K, Spittler A, et al. (2001) Oral feeding with glutamine prevents lymphocyte and glutathione depletion of Peyer's patches in endotoxemic mice. Ann Surg 234, 92-97.

40. Briassoulis G, Venkataraman S \& Thompson A (2010) Cytokines and metabolic patterns in pediatric patients with critical illness. Clin Dev Immunol 2010, 354047.

41. Träger K, DeBacker D \& Radermacher P (2003) Metabolic alterations in sepsis and vasoactive drug-related metabolic effects. Curr Opin Crit Care 9, 271-278.

42. Boelens PG, Nijveldt RJ, Houdijk AP, et al. (2001) Glutamine alimentation in catabolic state. J Nutr 131, Suppl. 9, 2569S-2577S

43. Karinch AM, Pan M, Lin CM, et al. (2001) Glutamine metabolism in sepsis and infection. J Nutr 131, Suppl. 9, 2535S-2538s.

44. Holecek M \& Sispera L (2014) Glutamine deficiency in extracellular fluid exerts adverse effects on protein and amino acid metabolism in skeletal muscle of healthy, laparotomized, and septic rats. Amino Acids 46, 1377-1384.

45. Roth E (2008) Nonnutritive effects of glutamine. J Nutr 138, 2025S-2031S

46. Hotchkiss RS, Tinsley KW, Swanson PE, et al. (2001) Sepsis-induced apoptosis causes progressive profound depletion of $\mathrm{B}$ and $\mathrm{CD}^{+}{ }^{+} \mathrm{T}$ lymphocytes in humans. J Immunol 166, 6952-6963.

47. Wesche DE, Lomas-Neira JL, Perl M, et al. (2005) Leukocyte apoptosis and its significance in sepsis and shock. $J$ Leukoc Biol 78, 325-337.

48. Luan YY, Yao YM, Xiao XZ, et al. (2015) Insights into the apoptotic death of immune cells in sepsis. $J$ Interferon Cytokine Res 35, 17-22.

49. Yin HY, Wei JR, Zhang R, et al. (2014) Effect of glutamine on caspase- 3 mRNA and protein expression in the myocardium of rats with sepsis. Am J Med Sci 348, 315-318.

50. de Oliveira GP, Silva JD, de Araújo CC, et al. (2014) Intravenous glutamine administration reduces lung and distal organ injury in malnourished rats with sepsis. Shock 41, 222-232.

51. Kim YS, Jung MH, Choi MY, et al. (2009) Glutamine attenuates tubular cell apoptosis in acute kidney injury via inhibition of the c-Jun N-terminal kinase phosphorylation of 14-3-3. Crit Care Med 37, 2033-2044.

52. Uehara K, Takahashi T, Fujii H, et al. (2005) The lower intestinal tract-specific induction of heme oxygenase- 1 by glutamine protects against endotoxemic intestinal injury. Crit Care Med 33, 381-390

53. Margaritis VG, Filos KS, Michalaki MA, et al. (2005) Effect of oral glutamine administration on bacterial tanslocation, endotoxemia, liver and ileal morphology, and apoptosis in rats with obstructive jaundice. World J Surg 29, 1329-1334.

54. Kessel A, Toubi E, Pavlotzky E, et al. (2008) Treatment with glutamine is associated with down-regulation of Toll-like receptor- 4 and myeloid differentiation factor 88 expression and decrease in intestinal mucosal injury caused by lipopolysaccharide endotoxaemia in a rat. Clin Exp Immunol 151, 341-347.

55. Singleton KD, Beckey VE \& Wischmeyer PE (2005) Glutamine prevents activation of NF- $\mathrm{kB}$ and stress kinase pathways, attenuates inflammatory cytokine release, and prevents acute respiratory distress syndrome (ARDS) following sepsis. Shock 24, 583-589.

56. Singleton KD, Serkova N, Beckey VE, et al. (2005) Glutamine attenuates lung injury and improves survival after sepsis: role of enhanced heat shock protein expression. Crit Care Med 33, 1206-1213. 